\title{
ESTUDO DO TRATAMENTO DE ÁGUA CONTAMINADA COM PIGMENTO UTILIZANDO RESÍDUO DE GRAVIOLA COMO AGENTE ADSORVENTE
}

\author{
M. T. $\operatorname{COSTA}^{1}$, D. C. HENRIQUE ${ }^{1}$, R. L. de ALMEIDA ${ }^{1}$, L. MEILI ${ }^{1}$, S. H. V. \\ CARVALHO $^{1}$, J. I. SOLETTI ${ }^{1}$ e A. K. S. ABUD ${ }^{2}$ \\ ${ }^{1}$ Universidade Federal de Alagoas, Centro de Tecnologia \\ ${ }^{2}$ Universidade Federal de Sergipe, Departamento de Tecnologia de Alimentos \\ E-mail para contato: lucas.meili@ctec.ufal.br
}

\begin{abstract}
RESUMO
A crescente preocupação com o meio ambiente e o aumento na quantidade de efluentes gerados pelas diversas indústrias têm estimulado a busca por formas de tratamento. Nesta vertente, a adsorção utilizando resíduos agroindustriais tem se apresentado como um método em ascensão no tratamento da água contaminada por diferentes tipos de pigmentos. Dentre os pigmentos utilizados em indústrias têxtil, de plástico, de papel, couro, dentre outras, o azul de metileno, corante orgânico e catiônico, merece destaque. A utilização do resíduo de graviola no tratamento deste tipo de efluente se mostra uma alternativa econômica e eficaz, apresentando remoção acima de $90 \%$. Os experimentos foram realizados em banho finito promovendo a variação da concentração do corante (50/100/150/200 ppm), da agitação (60/110/160 rpm) e da massa de agente adsorvente $(0,1 / 0,25 / 0,5 / 0,75 \mathrm{~g})$. Os resultados foram ajustados aos modelos cinéticos de pseudo primeira ordem e pseudo segunda ordem, apresentando bons resultados, e às isotermas de Langmuir, Dubinin-Radushkevich, RedlichPeterson, Sips, Toth, Freundlich e Khan, nas quais o ajuste não foi satisfatório.
\end{abstract}

\section{INTRODUÇÃO}

O crescente aumento na produção e uso de corantes tem gerado uma maior quantidade de efluentes contendo estes pigmentos, o que vem provocando a preocupação com a proteção do meio ambiente. Os efluentes gerados por indústrias têxteis, por exemplo, são descartados em rios e lagos, alterando a estabilidade biológica dos ecossistemas ali presentes (Sheng, Xie e Zhou, 2009).

Dentre estes corantes, o azul de metileno merece destaque. Este corante é comumente empregado na produção de papel e outros materiais como poliésteres e nylons, além da pigmentação de algodão, madeira e seda (Deng et al., 2011). Diante de suas características, o azul de metileno torna-se um resíduo difícil de tratar e, sendo catiônico, tem alta reatividade e capacidade de reagir com qualquer substrato (Poggere et al., 2011).

Uma vasta gama de tecnologias vem sendo empregada para o tratamento de efluentes, dentre elas a adsorção merece destaca. Trata-se de um fenômeno de superfície decorrente da aderência de moléculas de um componente presente em 
uma fase fluida (líquida ou gasosa) sobre a superfície de um sólido. Este método oferece vantagens significativas como baixo custo, disponibilidade, facilidade de operação, adequabilidade e eficiência em comparação com outros métodos, especialmente do ponto de vista econômico e ambiental (Demirbas, 2009).

Um dos resíduos agroindustriais passíveis de utilização em processos de adsorção é o resíduo de graviola. A gravioleira tem seu cultivo disperso em diversos estados do Brasil, sendo a Bahia o maior produtor, com produtividade superior a 30 T/ha. A produção ocorre ao longo do ano, com pico de colheita entre junho e fevereiro (Freitas, 2012).

Este trabalho tem por objetivo realizar o estudo do tratamento de água contaminada com o pigmento azul de metileno utilizando o resíduo de graviola como agente adsorvente, realizando ajustes aos modelos cinéticos de pseudo primeira ordem, pseudo segunda ordem e às isotermas de Langmuir, DubininRadushkevich, Redlich-Peterson, Sips, Toth, Freundlich e Khan.

\section{METODOLOGIA}

\subsection{Caracterização físico-química}

O resíduo de graviola utilizado nos experimentos correspondeu a sementes, polpa e casca do fruto, após a retirada do suco. Antes da utilização como agente adsorvente, o resíduo passou por corte e sanitização em hipoclorito de sódio $100 \mathrm{ppm}$ por $15 \mathrm{~min}$, procedendo a secagem em estufa com circulação de ar a $50{ }^{\circ} \mathrm{C}$ até peso constante. Em seguida, o resíduo foi triturado em moinho de facas tipo Willye e selecionado em peneira de 30 mesh, resultando em partículas de $0,6 \mathrm{~mm}$.

Realizou-se a caracterização físicoquímica do resíduo, avaliando-se umidade, $\mathrm{pH}$, teor de cinzas, densidade aparente e a microscopia eletrônica de varredura. A análise da umidade foi feita aquecendo-se a amostra a $105^{\circ} \mathrm{C}$ em estufa e a pesagem após 8 horas, até se obter o peso constante. A avaliação do $\mathrm{pH}$ foi feita promovendo a agitação da amostra contendo 2 gramas de resíduo em 50 $\mathrm{mL}$ de água destilada por 10 minutos. Logo após, o pH foi medido através de um pHmetro. A determinação do teor de cinzas foi feita por incineração simples, que consiste no aquecimento da amostra seca em mufla elétrica à temperatura de $500^{\circ} \mathrm{C}$, durante quatro horas ou até a obtenção de cinza clara, indicando a ausência de matéria orgânica. A avaliação da densidade aparente foi realizada medindo o volume ocupado por uma quantidade em massa definida do resíduo. Para a microscopia eletrônica de varredura (MEV) do resíduo foi utilizado um microscópio eletrônico de varredura por emissão de campo (field emission SEM; Shimadzu SSX-550), buscando investigar a morfologia das amostras de resíduo.

\subsection{Procedimento experimental}

Os estudos de adsorção foram realizados pela técnica de banho finito, sendo os parâmetros avaliados da forma demonstrada na Tabela 1.

$\mathrm{O}$ material foi posto em contato com $100 \mathrm{~mL}$ de solução do corante, preparada a partir de uma determinada quantidade em massa de azul de metileno diluída em água destilada. O sistema foi agitado em uma incubadora shaker Solab, a $25^{\circ} \mathrm{C}$. Segundo Silva (2005), a adsorção do azul de metileno é mais eficaz quando realizada a esta temperatura, promovendo uma maior quantidade adsorvida. As amostras foram coletadas em intervalos de tempo de 5, 10, 30, 60, 120, 180 e 300 min. Para análise do teor de corante foi utilizado o espectrofotômetro $\mathrm{UV}-\mathrm{Vis}$, no comprimento de onda de $665 \mathrm{~nm}$. As amostras foram analisadas em triplicata. 
Tabela 1 - Variáveis avaliadas no processo de adsorção.

\begin{tabular}{cccc}
\hline Análise & $\begin{array}{c}\text { Massa de } \\
\text { adsorvente }(\mathbf{g})\end{array}$ & $\begin{array}{c}\text { Concentração } \\
\text { do corante } \\
(\mathbf{p p m})\end{array}$ & $\begin{array}{c}\text { Agitação } \\
(\mathbf{r p m})\end{array}$ \\
\hline 1 & $0,1 / 0,25 / 0,5 / 0,75$ & 100 & 110 \\
2 & 0,5 & $50 / 100 / 150 / 200$ & 110 \\
3 & 0,5 & 100 & $60 / 110 / 160$ \\
\hline
\end{tabular}

Fonte: Autor(2015).

Segundo Rocha et al. (2012), a quantidade adsorvida no tempo $\mathrm{t}, \mathrm{q}_{\mathrm{t}}\left(\mathrm{mg} \mathrm{g}^{-1}\right)$, pode ser calculada pela Equação 1, enquanto a porcentagem de azul removido, pela Equação 2.

$$
\begin{aligned}
& q_{t}=\frac{\left(C_{0}-C_{t}\right) V}{W} \\
& \% \operatorname{Re} \text { moção }=\frac{\left(C_{t}-C_{0}\right)}{C_{0}} .100
\end{aligned}
$$

onde $C_{t}\left(\mathrm{mg} \mathrm{L}^{-1}\right)$ é a concentração do azul de metileno na fase líquida em qualquer tempo, $C_{0}\left(\mathrm{mg} \mathrm{L}^{-1}\right)$ é a concentração inicial da solução, $V(\mathrm{~L})$ é o volume da solução e $W(\mathrm{~g})$ é a massa do agente adsorvente.

Os modelos para ajustar os dados da cinética de adsorção foram o de pseudo primeira ordem e o de pseudo segunda ordem (Lagergren, 1898; Ho e Mckay, 1998), representados pelas Equações 3 e 4.

$$
q_{t}=q_{e}\left[1-\exp \left(-k_{1} t\right)\right]
$$

$$
q_{t}=\frac{k_{2} q_{e}^{2} t}{1+k_{2} q_{e} t}
$$

onde $k_{1}\left(\min ^{-1}\right)$ e $k_{2}\left(\mathrm{~g} \mathrm{mg}^{-1} \min ^{-1}\right)$ são constantes de pseudo primeira ordem e pseudo segunda ordem, respectivamente, $q_{t}$ $\left(\mathrm{mg} \mathrm{g}^{-1}\right)$ é quantidade adsorvida no tempo $t \mathrm{e}$ $q_{e}\left(\mathrm{mg} \mathrm{g}^{-1}\right)$, a quantidade adsorvida no equilíbrio.

Os ajustes foram realizados às isotermas de Langmuir, Dubinin-
Radushkevich, Redlich-Peterson, Sips, Toth, Freundlich e Khan, cujas equações encontram-se expressas na Tabela 2.

Tabela 2: Isotermas

\begin{tabular}{cc}
\hline Isotermas & Equações \\
\hline Langmuir & $q_{e}=\frac{Q_{0} b C_{e}}{1+b C_{e}}$ \\
Dubinin-Radushkevich & $q_{e}=q_{s} e^{-k_{a d} \varepsilon^{2}}$ \\
Redlich-Peterson & $q_{e}=\frac{k_{R} C_{e}}{1+a_{R} C_{e}^{g}}$ \\
Sips & $q_{e}=\frac{k_{S} C_{e}^{\beta_{S}}}{1+a_{S} C_{e}^{\beta_{S}}}$ \\
Toth & $q_{e}=\frac{k_{T} C_{e}}{\left(a_{S}+C_{e}\right)^{\frac{1}{t}}}$ \\
Freundlich & $q_{e}=k_{F} C_{e}^{1 / n_{F}}$ \\
Khan & $q_{e}=\frac{q_{S} b_{K} C_{e}}{\left(1+b_{K} C_{e}\right)^{a_{K}}}$
\end{tabular}

Fonte: Autor(2015).

\section{RESULTADOS E DISCUSSÕES}

Os resultados obtidos com a caracterização físico-química dos resíduos foram, de forma geral, favoráveis ao processo de adsorção. $\mathrm{O}$ valor de umidade encontrado, $10,90 \%$, caracteriza a pouca presença de água, promovendo o favorecimento do processo de adsorção devido à existência de poros livres para fixação das partículas de corantes. Com relação ao resultado obtido para o teor de cinzas, $1,60 \%$, observa-se que o resíduo de graviola é constituído principalmente de 
matéria orgânica, o que aumenta sua capacidade de adsorção. $\mathrm{O}$ pH 5,5 apresentado pelo resíduo demonstra que a adsorção ocorre em condições ácidas, o que, segundo Weber et al. (2014), favorece o processo adsortivo. Relativo à densidade aparente do resíduo, 0,383 $\mathrm{g} \mathrm{cm}^{-3}$, valores mais altos de densidade poderiam auxiliar na adsorção do fluido em sua superfície devido a uma maior área superficial de contato. Por sua vez, conforme Figura 1, a morfologia do resíduo apresenta uma superfície heterogênea, áspera e desigual, o que favorece o processo de adsorção do corante azul de metileno.

Figura 1 - Morfologia da superfície do resíduo de graviola

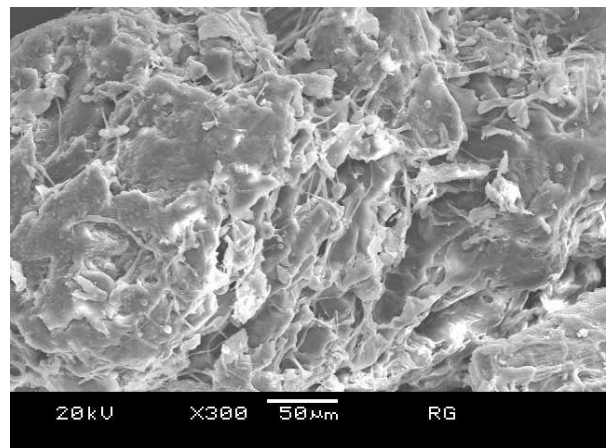

Fonte: Autor(2014).

Os resultados obtidos com a adsorção do azul de metileno após 300 minutos encontram-se demonstrados na Tabela 3.

Tabela 3 - Dados relativos ao processo de adsorção

\begin{tabular}{cc}
\hline Parâmetros & Remoção (\%) \\
\hline 50 ppm 0,5 g $110 \mathrm{rpm}$ & 66,66 \\
100 ppm 0,5 g $110 \mathrm{rpm}$ & 71,56 \\
150 ppm 0,5 g $110 \mathrm{rpm}$ & 83,44 \\
$200 \mathrm{ppm} \mathrm{0,5} \mathrm{g} 110 \mathrm{rpm}$ & 81,67 \\
100 ppm 0,1 g $110 \mathrm{rpm}$ & 59,00 \\
100 ppm 0,25 g $110 \mathrm{rpm}$ & 83,48 \\
100 ppm 0,75 g $110 \mathrm{rpm}$ & 91,65 \\
$100 \mathrm{ppm} \mathrm{0,5} \mathrm{g} 60 \mathrm{rpm}$ & 86,34 \\
100 ppm 0,5 g $160 \mathrm{rpm}$ & 89,36 \\
\hline
\end{tabular}

Fonte: Autor(2015).
É possível perceber que o resíduo de graviola apresentou ótimos valores de remoção do azul de metileno, apresentando uma remoção máxima acima de $90 \%$ para a concentração de corante 100 ppm, massa de adsorvente equivalente a $0,75 \mathrm{~g}$ e agitação de $110 \mathrm{rpm}$

Os resultados obtidos com a adsorção do azul de metileno foram ajustados aos modelos de pseudo primeira-ordem e pseudo segunda-ordem, sendo os valores encontrados demonstrados na Tabela 4.

Tabela 4 - Ajuste aos modelos de pseudo primeira-ordem e pseudo segunda-ordem

\begin{tabular}{|c|c|c|c|c|}
\hline \multicolumn{5}{|l|}{ Pseudo primeira ordem } \\
\hline Parâmetros & $q_{e}$ & $k_{l}$ & $\mathrm{R}^{2}$ & $\begin{array}{l}\text { ARE } \\
(\%)\end{array}$ \\
\hline $50 \mathrm{ppm} 0,5 \mathrm{~g} 110 \mathrm{rpm}$ & 5,050 & 0,152 & 0,788 & 8,098 \\
\hline 100 ppm 0,5 g 110 rpm & 13,980 & 0,003 & 0,943 & 0,929 \\
\hline 150 ppm 0,5 g $110 \mathrm{rpm}$ & 19,680 & 0,151 & 0,962 & 3,498 \\
\hline 200 ppm 0,5 g 110 rpm & 26,500 & 0,360 & 0,873 & 1,616 \\
\hline $100 \mathrm{ppm} \mathrm{0,1} \mathrm{g} 110 \mathrm{rpm}$ & 43,630 & 0,017 & 0,914 & 0,463 \\
\hline 100 ppm 0,25 g $110 \mathrm{rpm}$ & 29,930 & 0,027 & 0,906 & 0,916 \\
\hline 100 ppm 0,75 g $110 \mathrm{rpm}$ & 10,950 & 0,116 & 0,937 & 3,211 \\
\hline $100 \mathrm{ppm} 0,5 \mathrm{~g} 60 \mathrm{rpm}$ & 17,070 & 0,029 & 0,936 & 0,789 \\
\hline $100 \mathrm{ppm} 0,5 \mathrm{~g} 160 \mathrm{rpm}$ & 17,95 & 0,215 & 0,996 & 1,568 \\
\hline \multicolumn{5}{|l|}{ Pseudo segunda ordem } \\
\hline Parâmetros & $q_{e}$ & $k_{1}$ & $\mathrm{R}^{2}$ & $\begin{array}{c}\text { ARE } \\
(\%)\end{array}$ \\
\hline $50 \mathrm{ppm} 0,5 \mathrm{~g} 110 \mathrm{rpm}$ & 5,050 & 0,043 & 0,892 & 2,050 \\
\hline $100 \mathrm{ppm} \mathrm{0,5} \mathrm{g} 110 \mathrm{rpm}$ & 13,980 & 0,005 & 0,983 & 0,078 \\
\hline $150 \mathrm{ppm} 0,5 \mathrm{~g} 110 \mathrm{rpm}$ & 19,680 & 0,016 & 0,961 & 0,501 \\
\hline 200 ppm 0,5 g 110 rpm & 26,500 & 0,003 & 0,955 & 0,065 \\
\hline $100 \mathrm{ppm} \mathrm{0,1} \mathrm{g} 110 \mathrm{rpm}$ & 43,630 & 0,001 & 0,890 & 0,033 \\
\hline 100 ppm 0,25 g $110 \mathrm{rpm}$ & 29,930 & 0,002 & 0,974 & 0,033 \\
\hline $100 \mathrm{ppm} 0,75 \mathrm{~g} 110 \mathrm{rpm}$ & 10,950 & 0,017 & 0,984 & 0,291 \\
\hline $100 \mathrm{ppm} 0,5 \mathrm{~g} 60 \mathrm{rpm}$ & 17,070 & 0,003 & 0,976 & 0,062 \\
\hline $100 \mathrm{ppm} 0,5 \mathrm{~g} 160 \mathrm{rpm}$ & 17,950 & 0,026 & 0,999 & 0,129 \\
\hline
\end{tabular}

Através dos resultados obtidos, é possível observar um bom ajuste para a maioria das variações estudadas, apresentando 
coeficientes de determinação $\left(\mathrm{R}^{2}\right)$ satisfatórios, especialmente para o de pseudo segunda ordem, e médias de erro relativo (ARE) baixas.

Na Tabela 5, encontram-se os resultados obtidos com os ajustes às isotermas. Todos os ajustes apresentaram coeficientes de determinação em torno de $70 \%$, valor não satisfatório.

Tabela 5 - Isotermas, parâmetros e correlação obtidos no ajuste dos modelos.

\begin{tabular}{|c|c|}
\hline Isotermas & Parâmetros \\
\hline Langmuir & $\begin{array}{l}Q_{0}=43,63 \mathrm{mg} \mathrm{g}^{-1} \\
b=0,053 \mathrm{dm}^{3} \mathrm{mg}^{-1} \\
\mathrm{R}^{2}=0,713\end{array}$ \\
\hline Dubinin-Radushkevich & $\begin{array}{l}k_{a d}=-0,708 \mathrm{~mol}^{2} \mathrm{~kJ}^{-2} \\
\varepsilon=-0,258096 \\
\mathrm{R}^{2}=0,705\end{array}$ \\
\hline Redlich-Peterson & $\begin{array}{l}k_{R}=0,827 \mathrm{~L} \mathrm{~g}^{-1} \\
a_{R}=-0,241 \mathrm{mg}^{-1} \\
g=0,000044 \\
\mathrm{R}^{2}=0,702\end{array}$ \\
\hline Sips & $\begin{array}{l}k_{S}=0,605 \mathrm{~L} \mathrm{~g}^{-1} \\
a_{S}=0,024 \mathrm{~L} \mathrm{mg}^{-1} \\
\beta_{S}=-0,903 \\
\mathrm{R}^{2}=0,736\end{array}$ \\
\hline Toth & $\begin{array}{l}k_{T}=1,222 \mathrm{mg} \mathrm{g}^{-1} \\
a_{S}=0,000058 \mathrm{~L} \mathrm{mg}^{-1} \\
t=17,73 \\
\mathrm{R}^{2}=0,712\end{array}$ \\
\hline Freundlich & $\begin{array}{l}1 / n_{F}=1,416 \\
k_{F}=2,655 \mathrm{mg} \mathrm{g}^{-1} \\
\mathrm{R}^{2}=0,729\end{array}$ \\
\hline Khan & $\begin{array}{l}q_{s}=0,177 \mathrm{mg} \mathrm{g}^{-1} \\
b_{K}=46,302 \\
a_{K}=0,294 \\
\mathrm{R}^{2}=0,729\end{array}$ \\
\hline
\end{tabular}

Fonte: Autor(2015).

\section{CONCLUSÕES}

O resíduo de graviola apresentou-se um resíduo favorável ao processo de adsorção de pigmentos. A remoção máxima obtida foi superior a 90\%, indicando a eficiência do processo. Os resultados obtidos tiveram ajustes satisfatórios aos modelos de pseudo primeira ordem e pseudo segunda ordem; porém, os ajustes não foram eficientes às isotermas propostas.

\section{REFERÊNCIAS}

DEMIRBAS, A. Agricultural based activated carbons for the removal of dyes from aqueous solutions: a review. Journal of Hazardous Materials, 167, 1-9, 2009.

DENG，H.; LU，J.; LI，G.; ZHANG，G.; WANG, X. Adsorption of methylene blue on adsorbent materials produced from cotton stalk. Chemical Engineering Journal 172, 326 - 334, 2011.

FREITAS, A. L. G. E. Caracterização da produção e do mercado da graviola (Annona muricata L.) no Estado da Bahia. Dissertação. Universidade Estadual do Sudoeste da Bahia, 2012.

HO Y. S.; MCKAY G. Kinetic models for the sorption of dye from aqueous solution by wood. Process Safety and Environmental Protection, 76(B2), 183-191, 1998.

LAGERGREN S. About the theory of socalled adsorption of soluble substances. Kungliga Svenska Vetenskapsakademiens, 24(4), 1-39, 1898.

POGGERE, P.A.; DAVIS, R.; MONTANHER, S.F.; LOBO, V.S. Azul de Metileno: Propriedades e tratamentos. III ENDICT, UFTPR, Toledo, 2011.

ROCHA, O. O. S.; NASCIMENTO, G. E.; CAMPOS, N. F.; SILVA, V. L.; DUARTE, M. M. M. B. Avaliação do Processo Adsortivo Utilizando Mesocarpo de Coco Verde para Remoção do Corante Cinza Reativo BF-2R. Química Nova, v. 35, n. 7, 
São Paulo, 2012.

SHENG, J.; XIE, Y.; ZHOU, Y. Adsorption of methylene blue from aqueous solution on pyrophyllite, Appl. Clay Science, 46, 422 $-424,2009$.

SILVA, A. T. Influência da Temperatura na Adsorção do Corante Azul de Metileno Utilizando Serragem de Pinus elliotti como um Adsorvente Alternativo: Um Modelo para o Tratamento de Efluentes Têxteis. Trabalho de Conclusão de Curso, Universidade Federal de Santa Catarina, Florianópolis, 2005.

WEBER, C. T.; COLLAZZO, G. C.; MAZUTTI, M. A.; FOLETTO, E. L.; DOTTO, G. L. Removal of hazardous pharmaceutical dyes by adsorption onto papaya seeds. Departament of Chemical Engineering, Federal University of Santa Maria, 2014.

\section{AGRADECIMENTOS}

Agradecemos ao $\mathrm{CNPq}$ pelo apoio à pesquisa. 